\title{
Editorial
}

\section{Valve prostheses and pregnancy}

\author{
CELIA OAKLEY \\ From the Department of Clinical Cardiology, Hammersmith Hospital, London
}

All patients with mechanical heart valves need long term treatment with anticoagulant drugs to prevent thromboembolism. Because the use of these drugs in pregnancy increases the risk of abortion or fetal damage management of pregnant women with valve prostheses is still controversial. Women with artificial heart valves may even be advised against pregnancy because of the "serious risks to both mother and child". 1

A hypercoagulable state exists in pregnancy when concentrations of clotting factors, platelet turnover, and viscosity are increased and fibrinolysis is diminished. These changes increase the risk of prosthetic valve thrombosis. ${ }^{2}$

Whenever possible childbearing should be completed before valve replacement. In general, patients with severe mitral or aortic regurgitation tolerate pregnancy well probably because of the beneficial effects of vascular dilatation; the slight increase in heart rate seen in most pregnancies will limit aortic regurgitation. Patients with mitral stenosis suitable for valvotomy should have this carried out before pregnancy. For suitable valves closed mitral valvotomy during pregnancy can be successful without increased hazard to either mother or fetus if operation is avoided during the first trimester. This avoids prolonged admission to hospital with the adverse effects on the marriage and also ensures that the mother will be in good health to look after the infant and her family. Unfortunately, few surgeons in the developed world are now trained to do closed mitral valvotomy and risk of fetal loss when the operation is performed under bypass is considerable; however, many such operations have been performed successfully. Patients with mitral stenosis in sinus rhythm who are unsuitable for valvotomy or who have severe aortic stenosis (fortunately a rare condition) may be helped through pregnancy with $\beta$ blockers.

Requests for reprints to Dr Celia Oakley, Department of Medicine (Clinical Cardiology), Hammersmith Hospital, Ducane Road, London W12 0HS.
Fitness for pregnancy depends on the woman's general health and haemodynamic reserve; the reason for the valve replacement (such as Marfan's syndrome with its special maternal and genetic risk); the possible effects on fetal development of drugs given to the mother; the risk of thromboembolism, arrhythmia, or infection; and the maternal prognosis. Most patients with mitral or aortic valve replacements, even double or triple valve replacements for rheumatic heart disease, tolerate pregnancy well. Most such young patients are in New York Heart Association classes I or II, but a few may be in class III or IV and will be at increased risk. A full echocardiographic study should be carried out to evaluate left ventricular and valvar function and consolidate the clinical assessment. When there is doubt, an exercise test provides a valuable indication of the cardiac reserve.

Anaemia caused by menorrhagia is common when anticoagulants are being taken, and mechanical haemolysis may add to the risk of anaemia-although this is rare with modern valves unless there is a paraprosthetic leak. Patients with rheumatic valve disease may develop atrial fibrillation in pregnancy and if this occurs the rate should be brought under rapid control with digoxin and a $\beta$ blocker. Cardioversion can be carried out in pregnancy and does not induce ventricular fibrillation in the fetus. Other antiarrhythmic drugs that can be used safely are verapamil, quinidine, and disopyramide. If a diuretic is needed the dose should be kept to a minimum and adjusted to the pulmonary rather than the systemic venous pressure. Patients with mitral valve prostheses tend to have a raised venous pressure in pregnancy, particularly if atrial fibrillation is present.

Because rheumatic fever is now rare in developed countries, experience of large numbers of pregnancies in women with artificial heart valves is more often reported from the Third World than from the West. ${ }^{13-5}$ Most of the patients have mitral valve prostheses and just over a third of such patients in a recent report from Mexico were in atrial fibrillation. ${ }^{1}$ 
In the early years of experience with porcine xenografts and other "tissue" valves, their use was advocated in young women who might wish to become pregnant. ${ }^{6}$ Unfortunately, the long term durability of these valves is disappointing, particularly in the young ${ }^{7}$ and valve deterioration is likely to be especially rapid in pregnancy. The certain need for reoperation and the risk of between $5 \%$ and $10 \%$ for each operation make the use of tissue valves in the young unjustifiable. In any case the commonest condition requiring valve replacement in young women is rheumatic mitral valve disease in which an enlarged left atrium, previous thromboembolism, or atrial fibrillation impose an absolute need for long term anticoagulant treatment whatever kind of valve is implanted.

Coumarin derivatives carry a teratogenic risk of chondrodysplasia with nasal hypoplasia, optic atrophy, and dwarfism when they are administered between the sixth and ninth week of gestation. ${ }^{1489}$ The fetus becomes overanticoagulated because warfarin but not vitamin $\mathrm{K}$ dependent coagulation factors cross the placenta and the immature fetal liver is more sensitive to the anti-vitamin $\mathrm{K}$ effect of the drug. Neurological complications which have occurred after the administration of coumarin later on in pregnancy are almost certainly attributable to haemorrhage. This risk is further increased at delivery.

The antiplatelet agents, aspirin and dipyridamole, do not prevent thromboembolism from prosthetic valves in non-pregnant patients and they are even less effective during pregnancy when the mother is at increased risk of systemic embolisation. ${ }^{2410}$

Because heparin is a large molecule that does not cross the placenta and can have no teratogenic or anticoagulant effect on the fetus, it has been suggested for use during pregnancy either in prophylactic doses ${ }^{4}$ or for full anticoagulation employing a heparin lock, self-administration, and with the aim of achieving a partial thromboplastin time of $1.5-2.0 \times$ normal. ${ }^{11}$ The suggestion that heparin should be started before conception ${ }^{11}$ means that some women could remain on treatment indefinitely.

Hall et al reviewed 135 published cases in which heparin had been given during pregnancy to patients with artificial valves. ${ }^{12}$ The details of administration were not always clear but most were probably treated with subcutaneous heparin. Since either success or failure may stimulate reporting, such reviews may not give a true picture, but Hall found that an eighth of the infants were stillborn, a fifth were premature (a third of whom died), and only two thirds were normal-about the same success rate which followed the use of coumarin derivatives throughout pregnancy. Maternal haemorrhage occurred in $14(10 \%)$ of the patients and there were three deaths $(2 \%)$. The high fetal loss was attributable to retroplacental haemorrhage. Wang et al reported four abortions in 10 patients given subcutaneous heparin in the first trimester. ${ }^{13}$

As a compromise it has been suggested that the coumarins should be replaced by subcutaneous heparin during the first trimester and the last two weeks of pregnancy. An early report by Salazar et al of the use of heparin in a fixed dose of 5000 units every 12 hours was encouraging. ${ }^{4}$ More recently, his group examined the risk of embryopathy when coumarins were given throughout pregnancy, when heparin was given from the 6th to 12th week, and when heparin was started after the seventh week. The high incidence of spontaneous abortion was similar with all three regimens, but two women receiving subcutaneous heparin during the first trimester suffered massive valve thombosis and in another the valve thrombosed during the last two weeks of pregnancy after the patient had been transferred back to heparin.

Children were examined for signs of embryopathy by a clinical geneticist and this probably explains why the reported incidence was higher than in other reports. ${ }^{12} 14$ No abnormalities were seen in any of the children born after heparin had been used from the sixth week but two out of eight of the liveborn infants resulting from the 12 pregnancies treated with heparin between the 7th and 12th weeks had suggestive minor facial defects and two out of 27 children resulting from 37 pregnancies in women receiving coumarin drugs throughout pregnancy had features of embryopathy. No cases of central nervous system defects, dwarfism, or epiphyseal stippling were seen, however, so none of these children was seriously affected. Chen saw no cases of embryopathy among 22 pregnancies in which the mothers continued warfarin throughout the first trimester. ${ }^{3}$

None of the 54 children studied in the Mexican series had defects such as microcephaly or spasticity attributable to the use of coumarin anticoagulants during the last two trimesters of pregnancy. ${ }^{1}$

The sum of the recently reported experience suggests that a successful outcome can be expected in about two thirds ${ }^{6}$ of cases when coumarin derivatives are used throughout pregnancy. ${ }^{3612}$ The risk of a liveborn child having a disabling coumarin embryopathy seems to be $<5 \%$.

There are many published reports of valve thrombosis in mothers receiving heparin. ${ }^{13-17}$ This has occurred mainly, but not invariably in Björk-Shiley valves and usually after heparin prophylaxis, but there has been one report in a patient with a Starr 
mitral prosthesis who was on anticoagulant doses of heparin. ${ }^{16}$

The use of anticoagulant doses of heparin may reduce the risk of valve thrombosis (though this has still to be shown), but it carries a substantial risk of both maternal haemorrhage and spontaneous abortion. Moreover, it can also cause thrombocytopenia, alopecia, and osteoporosis. ${ }^{1718}$

Intravenous heparin has been widely used in hospital in the last two weeks of pregnancy without adverse effects. It has the virtue that both the mother and the fetus can be continuously observed. The effect of warfarin on the baby must be assumed to persist for seven to ten days after warfarin is stopped and the baby should be given vitamin $\mathrm{K}$. Breast feeding is not contraindicted. ${ }^{19}$. If the mother is admitted in premature labour while still on warfarin she should be given fresh frozen plasma before caesarean section. The effects of heparin can be temporarily reversed with protamine, and warfarin treatment can be resumed immediately after delivery.

It is uncertain whether the teratogenic effect of coumarin derivatives is the result of their chemical structure or of the anticoagulant effect exerted at a critical period of development of cartilagenous bone. The lack of information about the safety of phenindione during the first trimester may be attributable to its infrequent use rather than to its greater safety. Nevertheless, at Hammersmith Hospital we have for some years transferred young women to phenindione in pregnancy and have had no embryopathies and no compromised babies. ${ }^{20}$

Although both Hall and Salazar concluded that women of childbearing age with artificial valves should be advised not to attempt pregnancy, we are more optimistic ${ }^{2122}$ and believe that the danger to the mother is very small ${ }^{6}$; that the chance of a successful outcome is good; and that most unsuccessful pregnancies are miscarried so that the risk of a liveborn malformed baby is small. Whenever possible, childbearing should be completed before valve replacement, but when this is not possible, mechanical valves should be chosen and currently phenindione is the preferred anticoagulant with transfer to intravenous heparin in hospital during the last two weeks of pregnancy.

\section{References}

1 Iturbe-Alessio I, Fonseca M, Mutchinik O, Santos MA, Zajarias A, Salazar E. Risks of anticoagulant therapy in pregnant women with artificial heart valves. $N$ Engl J Med 1986;315:1390-3.

2 Schaffer AT. The hypercoagulable states. Ann Intern Med 1985;102:814-28.

3 Chen WWC, Chan CS, Lee PK, Wang RYC, Wong
VCW. Pregnancy in patients with prosthetic heart valves: an experience with 45 pregnancies. $Q \mathrm{~J} \mathrm{Med}$ 1982;51:358-65.

4 Salazar E, Zajarias A, Gutierrez N, Iturbe I. The problem of cardiac valve prostheses, anticoagulants and pregnancy. Circulation 1984;70 (suppl 1):1.169-77.

5 Ben Ismail M, Abid F, Trabelsi S, Taktak M, Fekih M. Cardiac valve prostheses, anticoagulation, and pregnancy. Br Heart $J$ 1986;55:101-5.

6 Oakley C, Doherty P. Pregnancy in patients after valve replacement. Br Heart J 1976;38:1140-8.

7 Magilligan DJ Jr, Lewis JW Jr, Tilley B. The porcine bioprosthetic valve. Twelve years later. $J$ Thorac Cardiovasc Surg 1985;89:499-507.

8 Ibarra-Perez C, Arevalo-Toledo N, de la Cadena AO, Noriea-Guerra L. The course of pregnancy in patients with artificial heart valves. $\mathrm{Am} \mathrm{J} \mathrm{Med}$ 1976;61:504-12.

9 Stevenson RE, Burton OM, Firlanto GS, Taylor HA. Hazards of oral anticoagulants during pregnancy. JAMA 1980;243:1549-51.

10 Biale Y, Cantos A, Lewen Thal H, Gueron M. The course of pregnancy: patients with artificial heart valves treated with dipyridamole. International Journal of Obstetrics and Gynaecology 1980;81:128-32.

11 Chesebro JH, Adams PC, Frister B. Antithrombotic therapy in patients with valvular heart disease and prosthestic heart valves. $\mathrm{J} \mathrm{Am}$ Coll Cardiol 1986;8:41-56B.

12 Hall JG, Paull RM, Wilson KM. Maternal and fetal sequelae of anticoagulation during pregnancy. $\mathrm{Am} \mathrm{J}$ Med 1980;68:122-40.

13 Wang RYG, Lee PK, Chow JF, Chen WWC. Efficacy of low dose, subcutaneous administered heparin in treatment of pregnant women with artificial heart valves. Med J Aust 1983;ii:126-8.

14 De Swiet M. Prescribing in pregnancy: anticoagulants. Br Med J 1987;294:428-30.

15 Larrea JL, Nunez L, Reque JA, Aguado M, Matarros R, Minguez JA. Pregnancy and mechanical valve prostheses: a high-risk situation for the mother and the fetus. Ann Thorac Surg 1983;459-63.

16 Bennett GG, Oakley CM. Pregnancy in a patients with a mitral valve prosthesis. Lancet 1968;ii:616-9.

17 Chong BH, Pitney WR, Castaldi PA. Heparin induced thrombocytopaemia: association of thrombotic complications with heparin-dependent 1 of $G$ antibody that induces thromboxane synthesis and platelet aggregation. Lancet 1982;ii:1246-8.

18 De Swiet M, Dorrington Ward P, Fidler J, et al. Prolonged heparin therapy in pregnancy causes bone demineralization (heparin induced osteopemia). $\mathrm{Br} J$ Obstet Gynaecol 1983;10:1129-34.

19 Baty JD, Breckenridge A, Lewis PJ, et al. May mothers taking warfarin breast feed their infants? $\mathrm{Br} J$ Clin Pharmacol 1976;3:969.

20 Oakley CM, Hawkins DF. Pregnancy in patients with prosthetic heart valves. $\mathrm{Br}$ Med J 1983;287:358.

21 Anonymous. Anticoagulants and heart valve replacement in pregnancy. Br Med $J$ 1977;i:1047-8.

22 Oakley CM. Pregnancy in patients with prosthetic heart valves. $B r M e d ~ J 1983 ; 286: 1680-2$. 